\title{
Aporte a la superación de la pobreza extrema: El liderazgo, una conquista en la agencia de la mujer
}

\author{
Juliana Arenas Gómez ${ }^{1}$
}

Ertilia Herrera Herrera ${ }^{2}$

\section{RESUMEN}

El presente artículo, es resultado del trabajo investigativo desarrollado con madres líderes del Programa Más Familias en Acción- Medellín Solidaria, pertenecientes a la comuna 13- San Javier de la ciudad de Medellín. El cual, tuvo por objetivo realizar un acercamiento a los significados construidos por éstas frente a su rol y como lo anterior contribuye a la superación de la pobreza extrema, posibilitando así, la generación de reflexiones que permitieran consolidar y afianzar cada vez más el acompañamiento que se viene realizando con cada una de ellas. Lo anterior, permitió identificar como el liderazgo construido por las mujeres está relacionado con su capacidad para creer, dirigirse, auto-liderarse y ser agentes de su propio desarrollo. Simultáneamente, estos aspectos, les permiten ir confluyendo procesos organizativos y comunitarios en sus territorios, buscando transmitir y transformar desde las necesidades evidenciadas en sus propios hogares, pero también desde el interés por construir maneras de encuentro alrededor de espacios que son comunes y de descubrir potencialidades en dicho camino.

Palabras clave: Desarrollo humano, Liderazgo, comunidad, pobreza, agencia, capacidades.

Recibido: 10 de diciembre de 2014

Aceptado: 10 de marzo de 2015

1 Maestra en Gestión del Desarrollo Comunitario Psicóloga Universidad de San Buenaventura, Medellín. Correo Electrónico: julianaarenasgomez@gmail.com

2 Master en Planificación ambiental y gestión de recursos naturales, especialista en gestión del desarrollo comunitario y en docencia universitaria. Correo Electrónico: eherrerah06@yahoo.es 


\title{
Contribution to overcoming extreme poverty: leadership, a conquest in the agency of women
}

\begin{abstract}
This article is the result of research work developed with leader mothers form the program More Families in Action- Solidarity Medellin, belonging to the commune 13- San Javier de Medellin. Which, it aimed to make an approach to the meanings constructed by them against their role as the above contribute to overcoming extreme poverty, thus enabling the generation of reflections that allows to consolidate and to strengthen every time more the accompaniment that has been done with each of them. The above-mentioned allowed identifying how leadership built by women is related to their ability to believe, direct, self-lead and be agents of their own development. Simultaneously, these aspects allow them to gradually converge organizational and community processes in their territories, seeking to convey and transform from the needs evidenced in their own homes, but also from the interest in building ways of gathering around spaces that are common and to discover potentials in that way.
\end{abstract}

Keywords: Human Development, Leadership, community, poverty, agency capabilities. 


\section{INTRODUCCIÓN}

A nivel mundial se han venido generando estrategias que permitan un mundo más igualitario, solidario, con un crecimiento colectivo y que aporte a la construcción del desarrollo humano. De allí, las alianzas y acciones conjuntas entre los países que permitan direccionar sus esfuerzos (Objetivos del milenio); uno de estos, centrado en la Erradicación de la pobreza extrema y el hambre.

De acuerdo a investigaciones de desarrollo social en Colombia (2004), la pobreza es entendida desde: " $L a$ incapacidad de los individuos dentro de su grupo familiar, de alcanzar los logros básicos de la vida humana de una manera libre" (pág. 31), asociada a la carencia de condiciones materiales, capacidades, derechos, exclusión social y a la accesibilidad a bienes y servicios. Por el contrario, la pobreza extrema, se refiere a las "condiciones tan precarias y de miseria aguda, que la opción de elegir les está negada" a las personas (Pág 33). El método, que permite situar a las personas en uno u otro grupo, sí bien depende de las dotaciones iniciales y posibilidades de intercambio; para el primer caso es a través de la medición de los niveles de ingresos de una persona, denominado, línea de pobreza. Mientras, que la segunda, está dado bajo la línea de indigencia.

Según el informe entregado por el Departamento Administrativo Nacional de Estadística, en el año 2012 a nivel Nacional el porcentaje de personas en situación de pobreza en Colombia fue de 32.7\%; mientras que un $10.4 \%$, se encontraba en pobreza extrema. Evidenciando que estos hogares tienen una tendencia a ser más numerosos; en su mayoría, de jefatura femenina, el nivel educativo es menor, reflejado en el analfabetismo y bajo acceso a la educación superior; sumado al desempleo y a las condiciones habitacionales en las que se encuentran (Núñez, J y Cuesta, L, 2006).
En la actualidad, en Colombia existe el Departamento para la Prosperidad Social, éste es una Entidad del Gobierno Nacional, que tiene como reto principal avanzar en la superación de la pobreza, la inclusión de la población vulnerable y víctima de la violencia, y la consolidación de los territorios a través de la garantía de la presencia del Estado en una senda de prosperidad y reconciliación. Algunos de los programas diseñados para el logro de lo anterior son: Más Familias en Acción, Ingreso para la Prosperidad Social, Jóvenes en Acción, Familias en su Tierra, entre otros.

En este marco general, se encuentra la iniciativa de cada departamento y municipio, quienes de acuerdo a las necesidades y características de cada territorio, buscan alianzas y articulaciones que les permita contribuir a los objetivos acordados.

En total, Colombia está organizada en 32 departamentos, uno de ellos Antioquia, el cual cuenta como ciudad principal Medellín, compuesta a su vez, por cinco corregimientos y 16 comunas, una de ellas San Javier- comuna 13. En ésta, se ha dado continuidad a acciones que permitan superar las situaciones de pobreza extrema e inequidad social, para lo que se han venido implementando diferentes programas que permitan contribuir a lo antes mencionado.

Medellín Solidaria, es un programa de la Alcaldía de Medellín desde donde se busca avanzar en la superación de la pobreza extrema a través del mejoramiento de la calidad de vida de los hogares más vulnerables de la ciudad; promoviendo su inclusión, autonomía social y económica en el marco de las nueve dimensiones del desarrollo humano. Para lo cual, realiza un trabajo articulado con entidades públicas y privadas que permitan la generación de oportunidades y el acceso integral y sostenible a la población.

Una de las principales articulaciones, es el Programa Más Familias en Acción, éste opera a través de 
transferencias condicionadas monetarias, buscando contribuir a la reducción de la pobreza y la desigualdad de ingresos, a la formación de capital humano y al mejoramiento de las condiciones de vida de las familias pobres y vulnerables mediante un complemento al ingreso. Además, de buscar obtener beneficios gracias a los espacios de participación comunitarios y el papel activo que desempeña la mujer en el programa (...) (Página Web Más Familias en Acción)

El programa Más Familias en Acción, se suma a la mayoría de los programas en América Latina que tienen un componente dirigido a combatir los problemas relacionados con el buen estado de salud, nutrición, educación de los menores de dieciocho años y fortalecer el tejido social; mediante procesos de encuentro e integración comunitaria. Lo anterior, bajo un principio de corresponsabilidad donde las familias son vistas como sujetos activos, responsables ante la sociedad y de ellas dependerá mejorar las condiciones de vida de sus hogares; de allí, que alterno, al pago del incentivo se promueven espacios de bienestar comunitario para que los beneficiarios participen de acciones que apunten al fortalecimiento del tejido social. Éste último proceso, se desarrolla a través de la figura de las madres o padres líderes quienes son titulares del programa, elegidos por los demás beneficiarios, de acuerdo al contexto, posibilidades, tiempo, directrices institucionales, entre otros; para encabezar los procesos de participación social y concertación y concertación con las madres de su entorno.

Diferentes investigaciones y evaluaciones del programa a corto y largo plazo han dado a conocer sus aportes frente a la superación de la pobreza, evidenciando que el incentivo contribuye a aminorar necesidades tales como alimentación, compra de útiles escolares, calzado y ropa, mejoras en la vivienda, pago de servicios y la vivienda (compra o arriendo) y creación de microempresa.
Adicionalmente, otros acercamientos han dado cuenta de como los espacios generados por las madres y padres líderes con sus grupos de titulares, han tenido efecto no sólo en la nutrición de los menores, a partir de conversaciones sobre el tema, sino que ha permitido aprender sobre diferentes tópicos de interés para quienes asisten, mejorando las relaciones familiares y sociales entre vecinos.

De allí, el interés por acercarse a estos liderazgos específicamente los desarrollados en la comuna 13San Javier, teniendo en cuenta la historia del contexto, la manera en la que emergen y como cada una de las líderes ha ido desarrollado no sólo tareas asignadas por el programa, sino que han generado unas prácticas propias, actividades, y ritmos de encuentro con la comunidad. Sumado, a un interés por conocer y acercarse a la realidad como un todo integrado, dinámico y en donde convergen la naturaleza y subjetividades de los individuos, llevando a un despertar investigativo que refleje las diferentes aproximaciones a los fenómenos y sus múltiples maneras de comprenderlo.

\section{MATERIALES Y MÉTODOS}

La metodología de la investigación, implementó como estrategia metodológica, el estudio de caso. Eumelia, G (2001) refiere que el término caso se puede referenciar de manera "“micro" o "macro", a personas, eventos particulares, grupos, organizaciones o instituciones, a una comunidad o subcultura (...) Bajo esta estrategia pueden ser estudiados multiplicidad de fenómenos tales como creencias, interacciones, actitudes, entre muchos otros" (Pág 71).

El abordaje metodológico fue desde la investigación cualitativa, la cual, aborda realidades subjetivas e intersubjetivas, permitiendo un contacto directo con los diversos actores y escenarios, eliminando y apartando prejuicios y creencias. Su naturaleza es flexible, evolucionaría y recursiva. 
La teoría de interpretación, fue a partir de la Teoría Crítica, la cual considera que todos los sujetos del proceso son parte activa de éste, como lo es el caso de las líderes, a fin de tener una visión holística de la realidad, en donde todos los actores están involucrados de manera participativa y real. Ubicándolas también en su realidad histórica y contemplando los factores políticos, valores, costumbres, lenguaje, etc., inmersos, que le permite tener una posición crítica y reflexiva de la realidad, con el fin de mejorar la práctica.

El enfoque de la investigación se realizó desde la etnometodología, resaltando el interés por la intersubjetividad, la acción social y el lenguaje en su cotidianeidad, contemplando su permanente construcción y comprensión de los sujetos inmersos en ella.

Para la recolección de la información, en un primer momento se hizo la revisión de fuentes secundarias como diarios de campo, memorias e informes; más adelante se llevaron a cabo tres entrevistas con madres líderes de la comuna 13 de la ciudad de Medellín, complementada por diferentes técnicas como la observación y grupos nominales, estos últimos con una participación de 44 liderezas. Con esto, se estableció un proceso de discusión teniendo en cuenta los hallazgos recogidos durante el trabajo de campo, el referente teórico establecido y los objetivos planteados para el ejercicio investigativo.

\section{RESULTADOS Y DISCUSIÓN}

Los modelos explícitos y explicativos que guiaron la investigación, para el caso de la pobreza se tuvo en cuenta las teorías y propuestas de Amartya Sen; Martha Nussbaum y Parker, $\mathrm{C}$ que acogen una postura multidimensional frente a ésta, tienen en cuenta el contexto, la privación de libertades y capacidades y la agencia del individuo sobre su propia historia, que a su vez, tiene injerencia en su manera de ser en el mundo, vivir, empoderarse y tomar decisiones libremente.
Para el caso, de liderazgo se retomaron supuestos de Maritza Montero, quien contextualiza el liderazgo en el ámbito comunitario y Alejandra Massolo, quien reflexiona sobre el lugar de la mujer en los procesos de participación.

La construcción de significados de las madres líderes, deja entrever como ser líder de la propia vida y abrirse camino y son momentos que contribuyen a potenciar la agencia de la mujer.

Lo anterior, se refleja en las motivaciones para representar y gestar acciones a favor de las titulares del programa y comunidad, acompañadas de sentimientos como alegría y miedo.

Así mismo, se evidenció que la asamblea de elección, es un momento determinante y significativo para cada una, tiene un valor de iniciación en su rol y legitimización ante la comunidad.

Es importante tener en cuenta que algunas expresan haber sentido un interés desde antes por ser líderes, pero, en su mayoría no refieren sus liderazgos a un proceso anterior e incluso manifiestan nunca haber pensado en hacerlo. En ambos casos las titulares, vecinos y familiares son quienes impulsan y motivan para su postulación, nombrándoles sus cualidades para relacionarse con la comunidad, hablar en público y haciéndoles saber que ellas podrían ser una buena líder y que contarían con su apoyo.

Entre sus motivaciones, se encuentran la intención de ayudar a los otros, la importancia de conocer más sobre el programa y aprovecharlo para resolver dudas e inquietudes en la comunidad, tener aprendizajes y crecer más como personas. Pero principalmente, la intención de buscar de espacios propios para integrarse y dejarse permear por la compañía de otras personas se constituyó en un agente movilizador para cada una. En este sentido, se evidencia una necesidad de socialización hacia el mundo público, iniciando 
afinidades y voluntades en la "aspiración de nuevas experiencias de sociabilidad y participación en la esfera pública, adquirir autoestima y poder salir del encierro doméstico" (Massolo, A. 2003)

Dichas relaciones, aportan también a la configurando de la base del poder de la líder, la cual nombra Montero, M, entendiéndolo como la capacidad para sentirse confiada y segura. En este caso, las líderes identifican diferentes redes de apoyo, tales como, la comunidad, las personas que les brindan el acompañamiento desde el programa y las demás líderes; pero primordialmente la familia, en especial, el papel de los hijos, quienes se convierten en unas figuras valorativas, aprobando o rechazando su rol y participación.

Ser líder entonces, es una transformación también de los roles familiares que estas asumien al interior de sus hogares, lo cual les implica apropiarse de nuevos aspectos y reconfigurarlo al interior de sus contextos.

Los relatos de las líderes, tanto en las entrevistas, como en los grupos generados, se refieren a ese transitar por la búsqueda de nuevas oportunidades, en especial, abrirse camino hacia ellas mismas.

Tal y como lo plantea Vásquez, S (2010) "El liderazgo personal es la condición indispensable para un auténtico liderazgo hacia fuera, hacia la comunidad, la organización o partido político. Es decir el liderazgo debe iniciar con la persona al ser capaz de dirigir su propia vida” (Pág. 56).

Desde la teoría de las capacidades de Sen, A (2000) esto se relaciona con una búsqueda de bienestar que se comienza a despertar en ellas, “(...) aspectos que contribuyen positivamente a reforzar la voz y la agencia de las mujeres a través de su independencia y del aumento de su poder" (Pág. 236).

Las líderes comienzan a mostrarse entonces, como agentes transformadoras y posibilitadores de cambios en sus territorios. Se comienza a despertar en ellas una capacidad para defender, expresar ideas y pensar colectivamente, que sí bien es un proceso, es decir algunas líderes lo sienten o manifiestan con mayor fuerza que otras, identifican como un aspecto fundamental capacitarse, formarse $\mathrm{y}$ adquirir herramientas para saber transmitir, compartir aprendizajes, desenvolverse frente a la comunidad, trabajar en equipo ; lo cual ellas lo llaman como un sentido de compromiso y responsabilidad frente a su quehacer, despertando conciencia sobre transmitir y compartir aquello que van conociendo y construyendo.

Esta capacidad de filiación, de la cual habla Nussbaum, M, no es más que ese lugar de identificación que encuentra el individuo con el otro, para ponerse en su lugar, reconocerlo como sujeto con potencialidades y posibilidades, y desde el deseo por juntarse y realizar propuestas conjuntamente.

Por consiguiente, su liderazgo se expresa en una oportunidad social, permeada por valores y costumbres que facilitan a los individuos ir configurando su destino e irse ayudando mutuamente. En donde la fuerza individual se convierte en una fuerza social, evidenciada en la manera de apropiarse del liderazgo, la adquisición de herramientas para desenvolverse, el reconocimiento de las necesidades barriales e identificación de las formas de llevar a cabo su labor comunitaria. Y es precisamente, el reconocimiento de su territorio, lo que las lleva a apropiarse de una manera de hacer y ser, ampliando y comprendiendo sus lógicas para actuar sobre la realidad, y desde allí posibilitar transformaciones en diferentes dimensiones de la sociedad. Para ello, cada una ha ido buscando la manera de acercarse a la comunidad llevando a cabo estrategias y acciones colectivas unas enfocadas en transmitir información del programa, como la posibilidad de que los beneficiarios tengan acceso "a", mientras que otras están relacionadas con fortalecer las dinámicas familiares y comunitarias, ambas con la intención de 
vincular a diversos actores, tales como niños, jóvenes y adultos y poder compartir con ellos estos espacios. Algunas de estas son las reuniones, el voz a voz, encuentros generados espontáneamente en la calle, en un medio de transporte, en un recorrido por el barrio, pegando información para que la comunidad se pueda informar o porque las titulares vayan y las busquen en su vivienda o lugar de trabajo.

Por consiguiente, el rol de las madres líderes, fortalece el capital social, reflejado en la capacidad de una comunidad para emprender obras colectivas tras objetivos comunes, en la medida que promueve la generación de redes, confianza y la organización comunitaria. (Parker, C, 2003).

\section{CONCLUSIONES}

- Las personas tienen la capacidad para decidir sobre su propio camino, estas mujeres, eligen el liderazgo en una búsqueda propia de reconciliación con ellas mismas, surge de ese sentimiento de ser líder de la propia vida, en un creer de sus habilidades y la fuerza para ejercer un rol en lo público. En este sentido, el hecho de que estas hayan tomado la decisión libremente de emprender liderazgos, se nombren, reconozcan, expresen sus sentimientos y se proyecten, hace parte de la construcción de su liderazgo y se convierten en un avance para su propio desarrollo y bien-estar

- La vinculación al liderazgo está marcado por una seria de motivaciones y expectativas que las líderes tienen frente al proceso, estas refieren en sí mismas, la búsqueda de nuevas maneras de ser y hacer personal y comunitariamente. En este sentido, se evidenció como ayudar a los otros, conocer más sobre el programa, aprender nuevas cosas, brindar información y mejorar la calidad de vida son los ejes en la intención del proceso, los cuales se van fortaleciendo a medida que van teniendo mayor contacto con los otros y participan del proceso de formación brindado desde el programa. Esto ha ido más allá de las actividades descritas desde el programa Más Familias en Acción, donde las líderes no sólo representan a las titulares, sino que poco a poco han ido configurando su propio proceso.

- El papel de la familia y lo que representa para líder tiene injerencia en la construcción de significados sobre el liderazgo, en un primer momento porque son quienes aprueban o rechazan su participación en el proceso, pero también porque lo que viven allí les permite comprender las relaciones, necesidades, dinámicas y problemáticas familiares que viven las demás personas con quienes generan los encuentros comunitarios.

- Las líderes han ido avanzando en el reconocimiento y apropiación de su comunidad, interpretándola y acercándose a ésta constantemente. Es allí, donde transita la mayoría del tiempo, establecen relaciones con los otros e identifican necesidades e interés. Esto, les ha permitido intencionar su rol desde el deseo por transformar y actuar sobre sus realidades, reconociéndose como parte de un territorio y su historia y fortaleciendo las formas de encuentro que realizan tanto con los niños, jóvenes, como adultos; además de afianzar su capacidad para constituir y estructurar la acción colectiva.

En este orden de ideas, el contacto con el otro les permite ir configurando una identidad en común, contenida tanto por aspectos personales, características en las que se encuentran, como por las diferencias de unos con otros; a partir de espacios para la integración social y cultural en los cuales se manifiesta el colectivo y en donde cada uno comienza a asumirse protagonista de la propia historia y de la vida en común.

- Los encuentros posibilitados desde las madres líderes en sus comunidades, ponen en manifiesto la acción colectiva e iniciativas de cada una de estas, constituyéndose en estrategias que permiten 
la superación de la pobreza, en la medida que están centradas en las personas, en sus potencialidades, necesidades y buscan la formación de sujetos que reflexionen sobre su diario vivir y puedan emprender alternativas para su bien-estar. Es por ello, que toma fuerza la búsqueda de un bienestar social y de un mejoramiento de las condiciones de vida, que sobrepasa lo individual y se pone en manifiesto en un deseo comunitario.

- Sí bien, cuando se habla de pobreza no se puede dejar de lado los ingresos económicos del individuo, cuando ésta es comprendida más allá de lo anterior, se evidencia como el liderazgo de las madres líderes del programa Más Familias en acción tiene una acción en su propio bienestar que permite fortalecerlas como mujeres, pero también como agentes de movimientos sociales, en la búsqueda de unas mejores condiciones personales, familiares y sociales. De esta manera, la pobreza entonces dejará entonces de ser una línea, para ser vista desde su dinamismo y las situaciones que permean al individuo $\mathrm{y}$ hacen parte de su realidad.

- La pobreza ha sido concebida como la imposibilidad de las personas para la realización de las capacidades, no porque carezcan de ellas, sino por las privaciones y restricciones que éstas se han encontrado para el desarrollo de su agencia. En este orden de ideas, el proceso que se ha venido realizando desde el acompañamiento grupal les ha brindado una serie de herramientas conceptuales y experienciales, constituyéndose en un puente para que las líderes expandan sus capacidades, fortalezcan su cualidades en el trabajo comunitario, se sientan más seguras, con la confianza para ir adentrándose en su propio mundo, encuentren una red de apoyo que les facilite su crecimiento y les permita expresar su liderazgo y capacidad de agencia.

- Realizar el ejercicio investigativo escuchando a las líderes desde cómo ellas han venido sintiendo el proceso desde su experiencia y capacidad para visibilizarse así mismas como seres en búsqueda de transformaciones propias y sociales, fueron elementos enriquecedores y formadores para pensarse la manera de acompañarlas y de ser también agentes facilitadores.

\section{RECOMENDACIONES}

- La investigación, como una manera de continuar reflexionando sobre los fenómenos sociales y mejorar las prácticas en el quehacer, deberá ser un aspecto a potenciar desde el programa Más Familias en Acción, específicamente para el caso del equipo de acompañamiento grupal de la ciudad de Medellín donde puedan compartir sus experienciasy maneras de trabajo; a fin de fortalecer las estrategias que se vienen implementando, la comprensión sobre la realidad y generar nuevas rutas de trabajo de acuerdo a las particularidades, contextos y situaciones propias.

- Se hace necesario continuar con el fortalecimiento y potenciación de las capacidades en las líderes, relacionado con el reconocimiento y lugar que se le brinda a cada una en la sociedad, no como sujetos pasivos, ni receptores, sino desde la creencia en la fuerza individual y social y desde un interés por ser agentes de participación. Para ello, es importante mayor acompañamiento desde el Programa Más Familias en Acción a nivel Nacional, desde donde las líderes también se puedan sentir acompañadas, escuchadas y apoyadas en su proceso.

- A fin de potenciar el acompañamiento brindado a las líderes, es importante revisar como los procesos de participación comunitaria que se vienen adelantando en cada uno de los territorios también influyen en la reconfiguración del liderazgo motivado desde el programa.

- La articulación con otras instituciones, organizaciones, proyectos y las madres líderes deberá ser ese paso hacia la construcción de un trabajo en red, a fin de generar acciones articuladas en el tiempo y promueva su accionar comunitario. 
- Es importante replantearse la transversalización del eje de género, desde una perspectiva de desarrollo centrada en la comunidad, inclusiva, planteamientos como el de Martha Naussman vienen brindan una salida más consensuadora y negociadora a los conflictos originados por las diferencias de sexo. Fortalecer el liderazgo desde las teorías de las capacidades con todo lo que ella implica es una propuesta que surge producto de este estudio.

\section{REFERENCIAS}

Comunicado de Prensa: Pobreza en Colombia. DANE (18 de Abril de 2013). Recuperado el 02 de Agosto del 2013 en: http://www.dane.gov.co/files/ investigacion es/condiciones_vida/pobreza/cp_ pobreza_2012.pdf

Cuadernos del Programa de las Naciones UnidasMinisterio de la protección Social. Investigaciones sobre desarrollo social en Colombia (2004) [Documento digital] http://www.pnud.org.co/img_ upload/9056f 18133669868e1cc381983d50faa/ cuaderno PNUDMPS2.pdf
Galeano (2001).Estrategias de investigación social cualitativa. Universidad de Antioquia. Facultad de Ciencias sociales y humanas.

Massolo, A (2003) El espacio local y las mujeres: pobreza, Participación y empoderamiento. Universidad Autónoma Metropolitana, México (UAM). Revista la Albaja

Núñez, J y Cuesta, L(2006). Evolución de las políticas contra la pobreza: de la previsión social a las transferencias condicionadas. Documento cede 2006-31 ISSN 1657-7191

Parker, C. (2003) Nuevos enfoques sobre evaluación de impacto para programas de superación de pobreza. CEPAL. Seminario taller: capital social, herramienta Para los programas de superación De la pobreza urbana y rural (edición electrónica) Programa Más Familias en Acción. Página web http://www.dps.gov.co/Ingreso_Social/Fa miliasenAccion.aspx

Sen, A (2000) Desarrollo y Libertad. Capitulo 1: La Perspectiva de la libertad. Buenos Aires, ed. Planeta S.A, p. 29-53

Vásquez, S (2010) Cuaderno de Trabajo 7: mujeres y liderazgo. Guatemala, NDI, 2010 